\title{
Gynea - Schweizerische Arbeitsgemeinschaft für Kinder- und Jugendgynäkologie
}

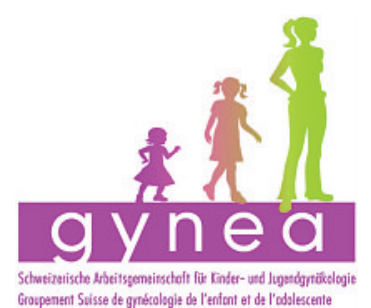

Korrespondenz:

Geschäftsstelle Gynea Noëlle Müller-Tscherrig Breitingerstrasse 23 CH-8002 Zürich Tel. 0794496206 gynea@gynea.ch

www.gynea.ch
Gynea vertritt die Anliegen der Kinder- und Jugendgynäkologie in der Schweiz. Der Verein (Schweizerische Arbeitsgemeinschaft für Kinder- und Jugendgynäkologie resp. Groupement Suisse de gynécologie de l'enfant et de l'adolescente) wurde 1992 in Montreux gegründet und ist seit 2005 ein Verein innerhalb der Schweizerischen Gynäkologischen Gesellschaft SGGG. Gynea hat zurzeit 127 Mitglieder und ist Mitglied der FIGIJ (Fédération Internationale de Gynécologie de l'Enfant et de l'Adolescente). Gynea ist international vernetzt, vor allem in Deutschland und Nordamerika. Der Vorstand besteht gleichermassen aus Gynäkologinnen sowie Kinder- und Jugendmedizinerinnen aus der gesamten Schweiz.

\section{Ziele von Gynea}

- Die Kinder- und Jugendgynäkologie füllt die Lücke, die in der Betreuung von gynäkologischen Erkrankungen, Symptomen, Fragestellungen und Problemen bei kleinen und heranwachsenden Mädchen besteht. Dabei spielt die interdisziplinäre Zusammenarbeit mit anderen Fachgebieten eine wesentliche Rolle.

- Die Arbeitsgemeinschaft fördert Kontakte und die Zusammenarbeit zwischen den Mitgliedern.

- Eine Aufgabe ist die Förderung von Forschung und Lehre im Bereich der Kinder- und Jugendgynäkologie, sowie der praktischen therapeutischen und präventiven Anwendung dieser Kenntnisse.

- Weiter- und Fortbildungsveranstaltungen sowie Kongresse werden organisiert.

- Ein weiteres Ziel ist die Etablierung von diagnostischen und therapeutischen Richtlinien und deren Vermittlung.

\section{Aktivitäten}

Gynea ist vor allem mit der deutschen Arbeitsgemeinschaft für Kinder- und Jugendgynäkologie vernetzt

\section{Der Gynea Vorstand}

Dr. med. Irène Dingeldein, FMH Gynäkologie und Geburtshilfe, Bern, Co-Präsidentin Dr. med. Renate Hürlimann, FMH Kinder- und Jugendmedizin, Zürich, Co-Präsidentin Dr. med. Saira-Christine Renteria, FMH Gynäkologie und Geburtshilfe, Lausanne, Wissenschaftliche Sekretärin

Dr. med. Marina Costa, FMH Kinder- und Jugendmedizin, Zürich

Dr. med. Ruth Draths, FMH Gynäkologie und Geburtshilfe, Luzern

Dr. med. Stefanie Eichenberger-Studer, FMH Kinder- und Jugendmedizin, Suhr

Dr. med. Christiane Kluckert, FMH Gynäkologie und Geburtshilfe, St. Gallen

Dr. med. Francesca Navratil, FMH Kinder- und Jugendmedizin, Zürich

Dr. med. Sibil Tschudin, FMH Gynäkologie und Geburtshilfe, Basel und empfiehlt deren lehrreiche Intensivkurse I und II in Kinder- und Jugendgynäkologie. Sie werden jedes Jahr im Sommer in Finsterbergen und im Herbst in München angeboten.

Gynea publiziert auf der Webseite alle Fortbildungen in der Schweiz, in Europa und Übersee (Veranstaltungen der NASPAG $=$ North American Society of Pediatric and Adolescent Gynecology). Die Vorstandsmitglieder halten regelmässig Vorträge zu aktuellen Themen, und auf der Homepage werden Fachartikel sowie Guidelines im Bereich der Kinder- und Jugendgynäkologie publiziert.

\section{Gynea Symposium}

Alle zwei Jahre organisiert Gynea ein Symposium. Das diesjährige Symposium findet am 17. September 2011 im Inselspital Bern statt. Neben Vorträgen werden nachmittags auch Workshops angeboten. Die Themen des Symposiums. sind unten aufgeführt. Weitere Informationen, z.B. zu den Referentinnen und Referenten sowie zur Anmeldung, finden sich unter www.gynea.ch.

\section{Gynea Symposium}

- Kinder- und Jugendgynäkologie seit 70 Jahren eine historische Betrachtung

- Varianten des äusseren weiblichen Genitales Wann sind weitere Abklärungen indiziert?

- Androgene - eine konstante Bedrohung für den weiblichen Phänotyp: Biochemische Grundlagen zur Diagnostik und Therapie

- Präsentation von zwei Fällen:

1. Wiederholte Episoden von Schmerzen im Beckenbereich bei einer weiblichen Jugendlichen 2. Vaginale Blutungen bei Kindern und Jugendlichen

- Die Vulva in der kinder- und jugendgynäkologischen Sprechstunde: Normal? Normvariante? Pathologie?

- Dermatologische Blickdiagnosen

- Sonographie - wann ist sie notwendig?

\section{Workshop-Themen:}

- Dermatologie + immer rot!

- Eine untergewichtige Jugendliche in der Praxis, die nicht richtig isst - wie weiter?

- Zyklus und Menstruation in der frühen Adoleszenz. Wann besteht Handlungsbedarf?

- Inkontinenz, Dysurie, Vulvitis, Ausfluss: wann, was, warum und wohin? Ein kinderchirurgischkindergynäkologischer Workshop. 